\title{
NEW UPPER BOUNDS FOR THE INFINITY NORM OF NEKRASOV MATRICES
}

\author{
LEI GAO AND QILONG LIU
}

Abstract. Some new upper bounds for the infinity norm of the inverse of Nekrasov matrices are presented. It is shown that the new bounds are better than those given by Kolotilina (2013) and $\mathrm{Zhu}, \mathrm{Li}$ (2017). Numerical examples are given to illustrate the effectiveness of the derived results.

Mathematics subject classification (2010): 15A60, 15A45, 65F35.

Keywords and phrases: Infinity norm, Nekrasov matrices, $H$-matrices.

\section{REFERENCES}

[1] Z. Z. BAI AND D. R. WANG, Generalized matrix multisplitting relaxation methods and their convergence, Numer. Math. J. Chin. Univ. 2(1) 1993, 87-100.

[2] L. Cvetković, H-matrix theory vs. Eigenvalue localization, Numer. Algor. 42(3-4) 2006, 229-245.

[3] L. Cvetković, V. Kostić And K. DoroslovačKic, Max-norm bounds for the inverse of $S$ Nekrasov matrices, Appl. Math. Compu. 218(18) 2012, 9498-9503.

[4] L. Cvetković, P. F. DAI, K. DoroslovačKiC AND Y. T. LI, Infinity norm bounds for the inverse of Nekrasov matrices, Appl. Math. Compu. 219(10) 2013, 5020-5024.

[5] M. García-Esnaola And J. M. PeÑa, Error bounds for linear complementarity problems of Nekrasov matrices, Numer. Algor. 67(3) 2014, 655-667.

[6] J. G. HU, Estimates of $\left\|B^{-1} A\right\|_{\infty}$ and their applications, Math. Numer. Sin. 3 1982, 272-282.

[7] J. G. Hu, Scaling transformation and convergence of splittings of matrix, Math. Numer. Sin. 5 1983, $72-78$.

[8] L. Y. Kolotilina, On bounding inverse to Nekrasov matrices in the infinity norm, Zap. Nauchn. Sem. POMI. 419 2013, 111-120.

[9] J. LiU, J. Zhang, L. Zhou AND G. Tu, The Nekrasov diagonally dominant degree on the Schur complement of Nekrasov matrices and its applications, Appl. Math. Comput. 320 2018, 251-263.

[10] C. Q. Li AND Y. T. LI, Weakly chained diagonally dominant B-matrices and error bounds for linear complementarity problems, Numer. Algor. 73 (4) 2016, 985-998.

[11] C. Q. LI AND Y. T. LI, Note on error bounds for linear complementarity problems for B-matrices, Appl. Math. Lett. 57 2016, 108-113.

[12] C. Q. LI, P. F. DAI AND Y. T. LI, New error bounds for linear complementarity problems of Nekrasov matrices and B-Nekrasov matrices, Numer. Algor. 74(4) 2017, 997-1009.

[13] C. Q. LI, H. PEI, A. GAO AND Y. T. LI, Improvements on the infinity norm bound for the inverse of Nekrasov matrices, Numer. Algor. 71(3) 2016, 613-630.

[14] W. Li, On Nekrasov matrices, Linear Algebra Appl. 281 (1-3) 1998, 87-96.

[15] Q. Tuo, Numerical Methods for Judging Generalized Diagonally Dominant Matrices, Doctor thesis, Xiangtan University, 2011,(In chinese).

[16] J. M. VARAH, A lower bound for the smallest singular value of a matrix, Linear Algebra Appl. 11(1) 1975, 3-5.

[17] F. WANG AND D. SUN, New error bound for linear complementarity problems for B-matrices, Linear Multilinear A. 66(11) 2018, 2156-2167.

[18] Y. ZHU AND Y. T. LI, New estimates of the infinty norm bounds for the inverse of Nekrasov matrices, Journal of Yunnan University: Natrual Science Edition. 39 2017, $13-17$. 